\title{
Inter-zone constraint modifies the stress-strain response of the constituent layer in gradient structure
}

\author{
Yanfei Wang ${ }^{1}$, Yuntian $\mathrm{Zhu}^{2^{*}}$, Xiaolei $\mathrm{Wu}^{3}$, Yueguang $\mathrm{Wei}^{4}$ and Chongxiang Huang ${ }^{1^{*}}$
}

\begin{abstract}
How an individual constituent zone behaves during the deformation of a heterostructured metallic material is a fundamental issue for understanding heterostructure deformation, but it remains a challenge to experimentally observe it. Here we report a study on the stress-strain behavior of the nanostructured gradient layer (NGL) in an integrated gradient specimen that consists of a coarse-grained (CG) central layer sandwiched between two NGLs. Constraint from the CG central layer led to the formation of dense and dispersed stable strain bands (SBs) in the NGL, which regained dislocation hardening after initial recovery and grain coarsening. Consequently, the NGL exhibited a transient plateau of flow stress after yielding, and then regained extra strain hardening to achieve excellent uniform elongation. These unique behaviors are dramatically different from those of a freestanding NGL, indicating a fundamentally different deformation principle that is intrinsic to heterostructures, i.e., inter-zone constraint modifies the constitutive behavior of constituent zones.
\end{abstract}

Keywords: heterogeneous structure, nanostructure, stress-strain response, strain bands, strength and ductility

\section{INTRODUCTION}

From bamboos to sea shells, heterostructures have evolved in biological systems to make them strong and tough [1]. This has inspired researchers to design heterostructures in engineering materials such as metals for superior strength and toughness [2-5]. Heterostructured metals, which consist of heterogeneous zones with dramatic variation $(>100 \%)$ in mechanical properties [6], can enable a synergistic effect where the global properties are better than the weighted sum of those of their constituent zones [7-16].
During the deformation of a heterostructured material, strain incompatibility among constituent zones is expected to introduce mutual constraint between them [6]. For example, the heterogeneity in elastic limit renders elastic/plastic interaction at the yielding stage, and the heterogeneity in strain hardening capability leads to plastically stable/unstable constraint at a large strain stage due to the incompatibility in plastic stability $[8,17-19]$. Such inter-zone constraint is believed to be responsible for the synergistic mechanical responses [2,6-8,16-19]. This raises a question: is the deformation behavior/mechanism of constituent zones in a heterostructured material different from that of their freestanding counterparts? If yes, what is the difference between them, and what is the mechanism for the change? These questions are fundamental issues for understanding the deformation mechanism, optimizing the microstructural architecture, and predicting the mechanical properties in practical engineering design. For example, the actual constitutive property of constituent zones, rather than the behavior of their freestanding counterparts, is needed for evaluating the deformation of heterostructured materials using finite element simulation.

Inter-zone constraint changes their stress state and causes gradient strain distribution near the zone boundary [16-21]. Piling-up of geometrically necessary dislocations in the strain gradient zone will promote dislocation entanglement and the development of longrange internal stresses, which collectively result in extra hardening [7,22-29]. This latest insight sheds light on the integrated effects of inter-zone interaction. However, it has not yet been quantitatively observed how inter-zone constraint affects the mechanical behavior of constituent zones, due to the experimental difficulties in simulta-

\footnotetext{
${ }^{1}$ School of Aeronautics and Astronautics, Sichuan University, Chengdu 610065, China

${ }^{2}$ Department of Materials Science and Engineering, City University of Hong Kong, Hong Kong, China

${ }^{3}$ State Key Laboratory of Nonlinear Mechanics, Institute of Mechanics, Chinese Academy of Sciences, Beijing 100190, China

${ }^{4}$ Department of Mechanics and Engineering Science, College of Engineering, Peking University, Beijing 100871, China

* Corresponding authors (emails: chxhuang@scu.edu.cn (Huang C); y.zhu@cityu.edu.hk (Zhu Y))
} 
neous measurements of microscale stress and strain, and the theoretical difficulties in evaluating local synergistic effects.

Here we present an experimental investigation on the stress-strain response of a constituent layer in a gradient structure. It is found that the hetero-deformationinduced synergistic constraint from its neighboring layer changed its flow stress evolution, strain hardening behavior, uniform elongation and strain accommodation mechanism.

\section{EXPERIMENTAL SECTION}

\section{Preparation of gradient material}

A commercial pure $\mathrm{Ni}$ (99.6 wt.\%) sheet was selected as the raw material. After annealing at $750^{\circ} \mathrm{C}$ for $4 \mathrm{~h}$, the coarse-grained (CG) plate was treated by rotationally accelerated shot peening to produce the gradient structure, using the 1-mm-diameter steel balls at a controlled velocity of $40 \mathrm{~m} \mathrm{~s}^{-1}$ for a duration of $5 \mathrm{~min} \mathrm{[30].} \mathrm{To}$ smooth the surface, the gradient-structured plate was further treated using 0.5 -mm-diameter small balls at the same velocity but longer duration of $10 \mathrm{~min}$.

The microstructure gradient of the as-processed material was characterized using scanning electron microscopy (SEM) and transmission electron microscopy (TEM) (Fig. 1a-d). After tensile deformation, the TEM foils were lifted out from designated positions using focused ion beam. TEM observations were conducted in a JEM 2010 microscope at $200 \mathrm{kV}$. The hardness gradient of the asprocessed material was measured using a Vickers hardness tester, at a load of $25 \mathrm{~g}$ for $15 \mathrm{~s} \mathrm{(Fig.} \mathrm{1e).} \mathrm{Indentations}$ were arranged along a zigzag line, and the spacing be- tween neighboring indentations was three times longer than impression diagonal.

As shown (Fig. 1), the gradient material consists of two nanostructured gradient layers (NGLs) sandwiching a CG central layer. The thicknesses of NGL and homogeneous CG central layer are 0.76 and $2.08 \mathrm{~mm}$, respectively. Hardness gradient, from $\sim 265 \mathrm{Hv}$ in the nanostructured surface to $\sim 130 \mathrm{Hv}$ near the CG layer, exists in the NGL.

\section{Uniaxial tensile tests}

Fig. 2 illustrates the preparation process of tensile specimens. Dog-bone shaped tensile specimens with an integral gradient structure and a gauge dimension of $18 \mathrm{~mm} \times 4.9 \mathrm{~mm} \times 3.6 \mathrm{~mm}$ were machined from the gradient plate. After $x \%$ tensile prestrain, the NGL of the integrated gradient specimen was peeled off by polishing away other layers, and labeled as $\mathrm{NGL}_{x \%}$ tensile specimen. $\mathrm{NGL}_{0 \%}$ represents the freestanding NGL that has undergone $0 \%$ prestrain. During the polishing process, the thickness of $\mathrm{NGL}_{x \%}$ specimens was precisely controlled by referring to the center of prearranged indentations, which were arranged on the lateral surfaces of the integrated gradient specimens at an accurate depth of $0.76 \mathrm{~mm}$ before prestraining. Uniaxial tensile tests were performed at a nominal strain rate of $5 \times 10^{-4} \mathrm{~s}^{-1}$. An extensometer in gauge length of $12 \mathrm{~mm}$ was used to measure the strain during uniform elongation. The tests for each type of $\mathrm{NGL}_{x \%}$ sample were repeated on at least four independent specimens.

\section{Strain and height contour characterizations}

During tensile testing, the distribution and evolution of strains on the nanostructured surface were recorded by in
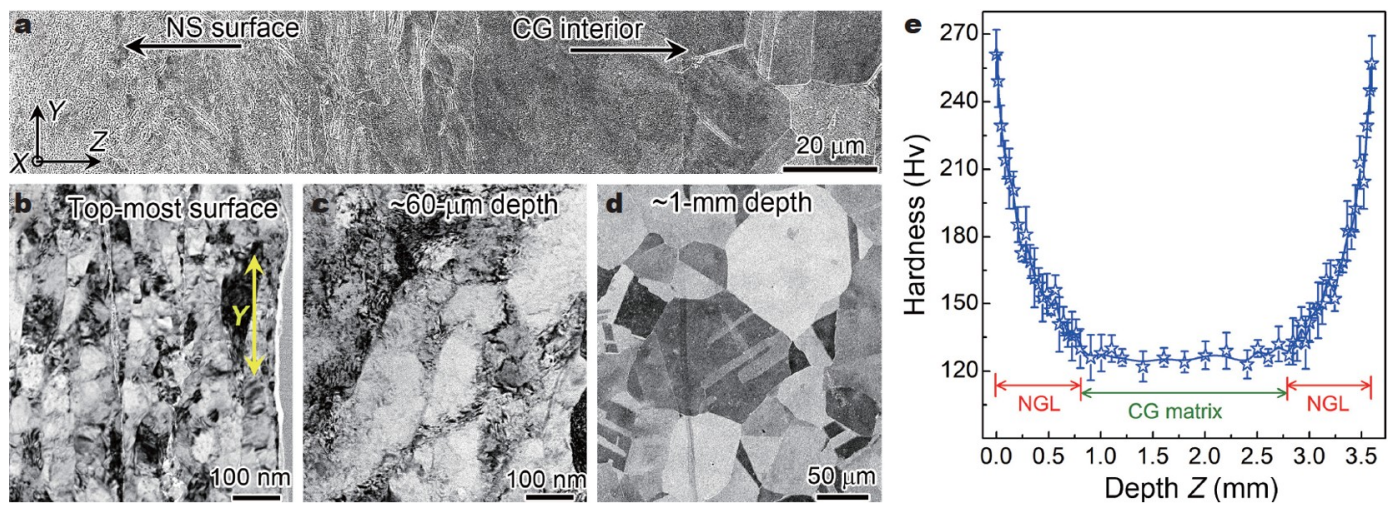

Figure 1 As-processed gradient structure. (a) A global SEM image showing the gradual transition from nanostructured surface to severely deformed subsurface layer (with obliterated initial grain boundary). Bright-field TEM images showing (b) the nanostructure (NS) at the topmost surface layer and (c) the immature NS with dense dislocation substructures at the depth of $\sim 60 \mu \mathrm{m}$. (d) An SEM image showing the equiaxed CG center. (e) Hardness profile showing the mechanical gradient along thickness, and illustrating the partitioning of NGL and CG center. For the coordinate in (a), $Z$ is the thickness direction, and $X Y$ plane is parallel to the peening surface. 


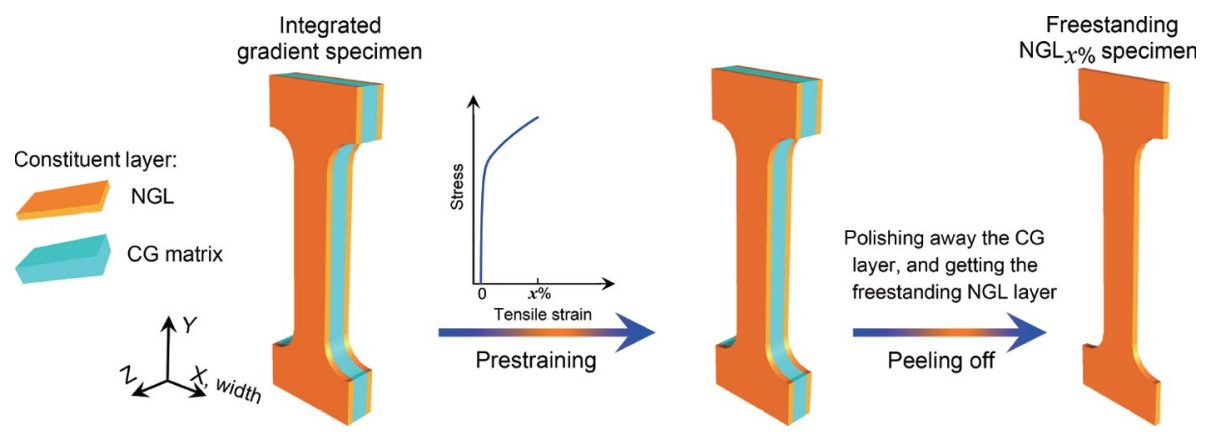

Figure 2 Schematic sketches illustrating the geometry, constituents and preparation process of tensile specimens. The coordinate is same as that in Fig. 1, where $Y$ is the tension direction.

situ digital image correlation (DIC) technique, using a short-focus optical lens. The height contour of characteristic strain bands (SBs) formed after the tensile test was measured using a white light interferometry, which has a height resolution as high as $\sim 20 \mathrm{~nm}$.

\section{RESULTS}

\section{Mechanical response of the NGL in gradient structure}

Fig. 3a shows the engineering stress-strain curves. The integrated gradient specimen (the longest solid curve) exhibits a superior strength-ductility combination, suggesting that the NGL deformed coherently with CG interior to a decent strain. Two interesting points can be drawn from the responses of $\mathrm{NGL}_{x \%}$ specimens (dashed curves).

First, the $\mathrm{NGL}_{x \%}$ specimen, which has experienced $x \%$ prestrain before being peeled off from the integrated gradient specimen, is still able to achieve decent uniform elongation (the shadowed region in Fig. 3b). For example, the $\mathrm{NGL}_{3.5 \%}$ specimen displays $16.1 \% \pm 0.8 \%$ uniform elongation, which is even larger than that of $\mathrm{NGL}_{0 \%}$ specimen $(11.7 \% \pm 1.2 \%)$. This implies that some type of unusual uniform straining mechanism might have been activated in the NGL during prestraining. Moreover, the larger the prestrain, is the larger the total uniform elongation was experienced by the $\mathrm{NGL}_{x \%}$ (the fitted red curve in Fig. 3b), confirming that the retention of the unusual ductile mechanism in the NGL was assisted by the CG central layer.

Second, when the prestrain $x \%$ is smaller than $~ 5 \%$, the prestraining has little effect on the yield strength of $\mathrm{NGL}_{x \%}$ specimens, while higher prestrains enable dramatic increase in the yield strength. For example, the prestrain-induced yield strength increment in $\mathrm{NGL}_{8 \%}$ and $\mathrm{NGL}_{16 \%}$ specimens is much higher than that in the $\mathrm{NGL}_{3.5 \%}$ specimen. The yield strength, and the flow stresses at $0.5 \%$ and $1 \%$ plastic strains $\left(\varepsilon_{\mathrm{p}}\right)$ of $\mathrm{NGL}_{x \%}$ specimens were extracted and fitted as a function of the corresponding total true strain (including the prestrain), which is plotted as the red curve in Fig. 3c.

The $\mathrm{NGL}_{x \%}$ specimen was no longer constrained by the CG layer when tested alone. However, during prestraining in the integrated gradient specimen, the constraint from CG layer affected its stress state and accumulation of crystal defects $[8,9,17]$. One can assume that before unloading the prestrained sample and after reloading the freestanding $\mathrm{NGL}_{x \%}$ specimen to yield, the relative change of microstructure (such as dislocation tangles) in NGL is negligible. The same assumption was made in the theoretical derivation of the hetero-deformation-induced stress from hysteresis loops [24,31,32]. Therefore, the fitted flow stress (the red curve in Fig. 3c) can be attributed to the unique defect structure of $\mathrm{NGL}_{x \%}$ specimens formed under the constraint of CG layer during prestraining. In other words, the fitted flow curve can be considered as an approximation of the stress-strain response of the NGL in an integrated gradient specimen, which is affected by the interaction with CG layer.

As shown, the fitted stress-strain curve (Fig. 3c) exhibits a transient flow plateau at the stage after yielding, causing a dramatic decrease, followed by a quick up-turn, in strain hardening rate $(\Theta=\mathrm{d} \sigma / \mathrm{d} \varepsilon$, Fig. 3d). Since the CG side of NGL should display stable strain hardening, the $\Theta$ up-turn implies that the NS side experienced significant strain softening first and then a recovery of strain hardening. At the higher-strain stage, in contrast to the quick instability of the freestanding $\mathrm{NGL}_{0 \%}$ specimen (the black curves in Fig. $3 c$ and d), the NGL in the integrated gradient specimen retained much higher strain hardening. These observations confirm that the actual stress-strain behavior displayed by the NGL in a gradient structure is significantly different from the performance of a freestanding NGL. 

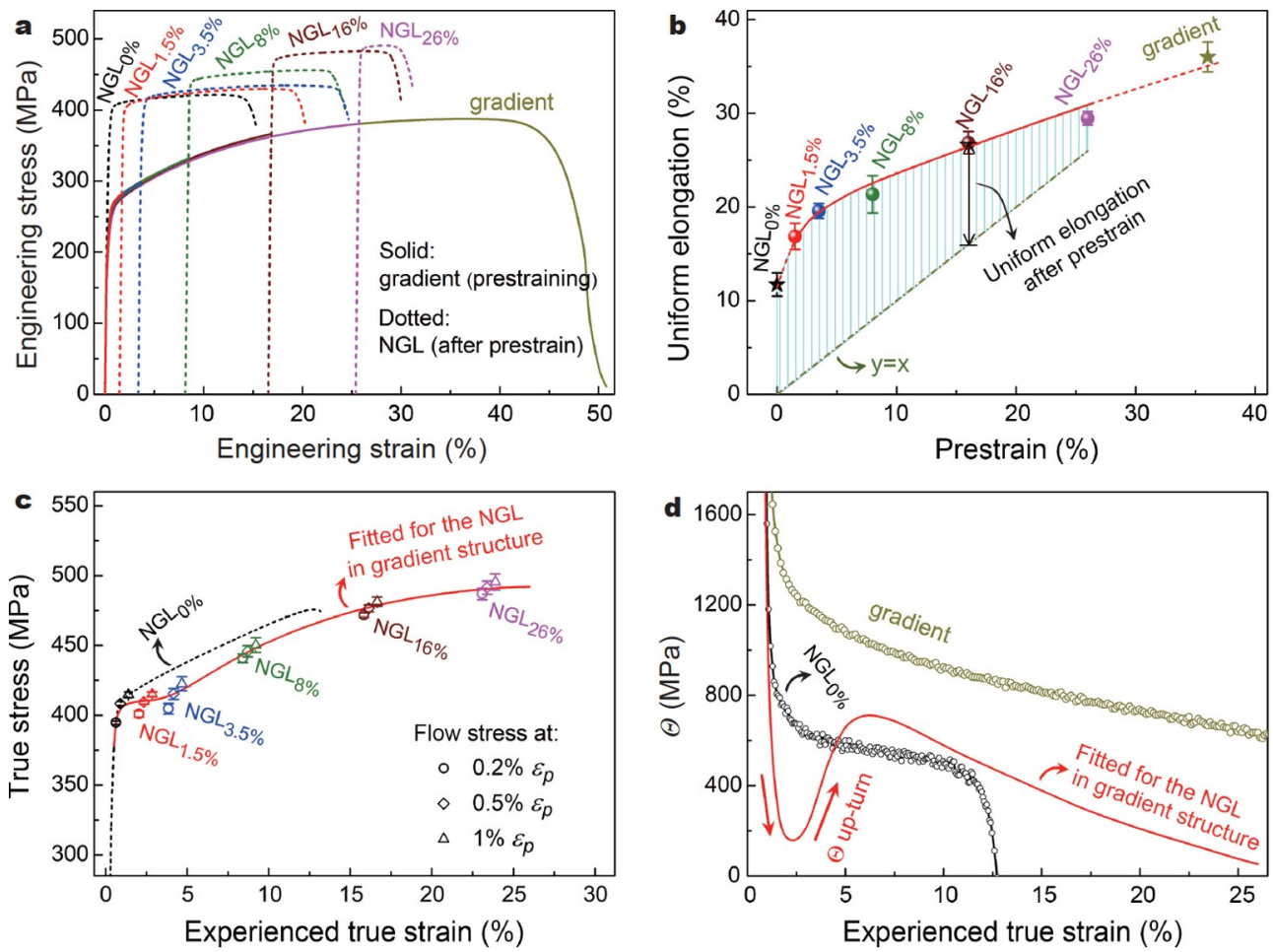

Figure 3 Stress-strain response of the NGL in the integrated gradient structure. (a) Uniaxial tensile stress-strain curves (dashed) for freestanding $\mathrm{NGL}_{x \%}$ specimens. The colored solid curves show the prestraining process of integrated gradient specimens. (b) Uniform elongation experienced by $\mathrm{NGL}_{x \%}$. Shadowed region shows the uniform elongation displayed after prestrain. (c) The stress-strain response of the NGL in the integrated gradient structure (the red solid curve). Tensile behavior of the freestanding $\mathrm{NGL}_{0 \%}$ specimen (the black dotted curve) is also shown for comparison. (d) Strain hardening responses, in which the red curve is derived from the fitted stress-strain response in (c).

\section{Dispersed SBs in the NGL of gradient structure}

The excellent uniform elongation achieved by the NGL in the integrated gradient specimen, which is almost twice that of the $\mathrm{NGL}_{0 \%}$ specimen (Fig. $3 \mathrm{c}$ ), can be primarily attributed to the development of dispersive SBs. In contrast to the quick coalesce of catastrophic strain localization in the $\mathrm{NGL}_{0 \%}$ specimen (Fig. 4a), dense stain bands are dispersively formed over the entire NS surface of the integrated gradient specimen (contours on the left side of symbol " + " in Fig. 4b and c), as partially indicated by white arrows. Fig. 5 presents the height contour across a representative mature SB. Such dispersed SBs sustain most of the applied strain, with none of them carrying high enough strain concentration to propagate across the whole cross-section. In other words, these dispersed SBs are mechanically stable, which leads to macroscopically homogeneous strain distribution in the NGL of the integrated gradient specimen. Since such dispersed SBs cannot form in the $\mathrm{NGL}_{0 \%}$ specimen, these observations also suggest that the CG interior should have played a critical role in activating them and maintaining their stability [30,33-35].

It is surprising that, as partially indicated by yellow arrows, dispersed SBs were also developed in the $\mathrm{NGL}_{x \%}$ specimens (maps on the right side of symbol " + " in Fig. $4 \mathrm{~b}$ and $\mathrm{c}$ ). The strain distributions across two representative SBs (Fig. 6a) are plotted in Fig. $6 \mathrm{~b}$ and c, which reveal that the SBs nucleated during prestraining can continue to accommodate the applied strain in the tension of freestanding $\mathrm{NGL}_{x \%}$ specimens. More interestingly, during the tensile straining of a freestanding $\mathrm{NGL}_{x \%}$ specimen, SBs increased quickly to reach a saturated density much higher than that of the $\mathrm{NGL}_{0 \%}$ specimen, but comparable to that on the surface of an integrated gradient specimen that had undergone $x \%$ strain (Fig. 6d). For example, the saturated density of SBs formed in the $\mathrm{NGL}_{1.5 \%}$ specimen is $\sim 1825 \mathrm{~m} \mathrm{~m}^{-2}$, which is comparable to the SB density $\left(\sim 1850 \mathrm{~m} \mathrm{~m}^{-2}\right)$ of an integrated gradient specimen that has just undergone $1.5 \%$ tensile strain. These observations indicate that the ductilization mechanism activated for the NGL in the integrated gradient specimen, i.e., dispersively stable strain 


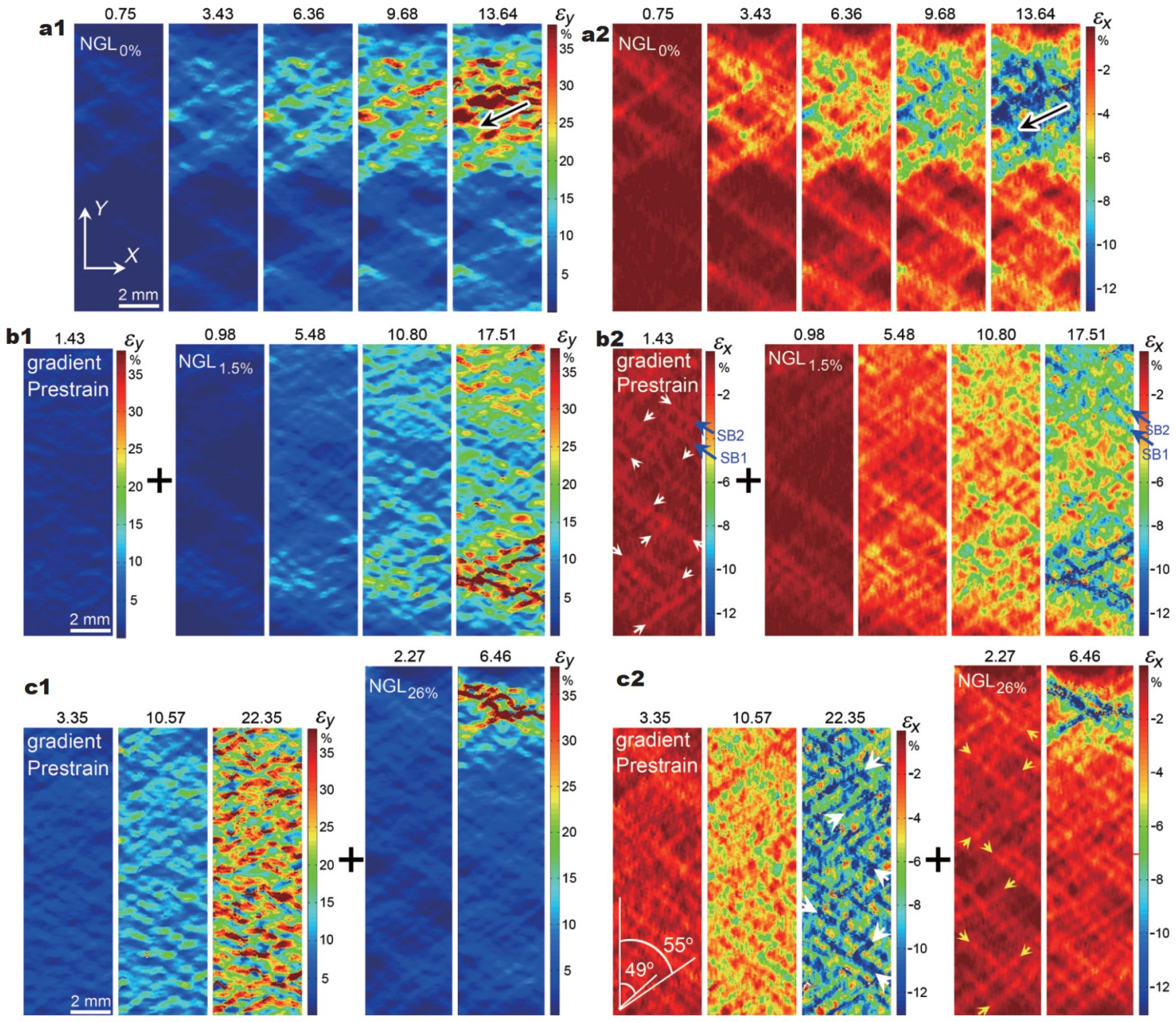

Figure 4 Strain maps measured on the nanostructured surfaces, showing the dispersed SBs developed in the NGL of the integrated gradient specimen. The tensile strain $\varepsilon_{y}(\mathrm{a} 1, \mathrm{~b} 1$ and $\mathrm{c} 1)$ and the corresponding shrinking strain $\varepsilon_{x}(\mathrm{a} 2, \mathrm{~b} 2$ and $\mathrm{c} 2)$ experienced by the $(\mathrm{a} 1, \mathrm{a} 2) \mathrm{NGL}_{0 \%},(\mathrm{~b} 1, \mathrm{~b} 2)$ $\mathrm{NGL}_{1.5 \%}$, and $(\mathrm{c} 1, \mathrm{c} 2) \mathrm{NGL}_{26 \%}$ specimens. The coordinate is same as that in Fig. 2. In (b1, b2) and (c1, c2), subgraphs on the left side and right side of symbol "+", respectively, were taken during the prestraining process of the integrated gradient specimen and during the tension of freestanding $\mathrm{NGL}_{x \%}$ specimen. SBs are the strain concentration bands orientated at $49^{\circ}-55^{\circ}$ with respect to the tensile axis (warm-colored in $\varepsilon_{y}$ contour and cold-colored in $\varepsilon_{x}$ contour). The number above each subgraph represents the applied tensile strain. The black arrow in (a) indicates the coalescence of strain localization. The white and yellow arrows in (b2) and (c2) mark a part of dispersed SBs.

banding, can be inherited by the freestanding $\mathrm{NGL}_{x \%}$ specimen. This is the reason why $\mathrm{NGL}_{x \%}$ specimens can still display decent uniform elongations (Fig. 3a and b).

\section{DISCUSSION}

Inter-layer constraints lead to the formation of dispersed SBs

During tensile straining of the integrated gradient specimen, local plastic instability can be activated at the stress concentration sites of the nanostructured surface layer soon after yielding, i.e., early strain concentration bands nucleation, due to its low strain hardening capability [3640]. However, for the strain banding region in the nanostructured surface layer, the localized strain is incompatible with the uniform straining behavior of the neighboring CG layer. Specifically, both the tensile strain $\varepsilon_{y}$ and the lateral shrinking strain $\varepsilon_{x}$ in the strain banding region are higher than that of the CG neighbor. Additional constraints, $\sigma_{y}^{-}$and $\sigma_{x}^{+}$are therefore introduced from the latter in order to coordinate their deformation as much as possible (Fig. 7a). This changes the stress state 

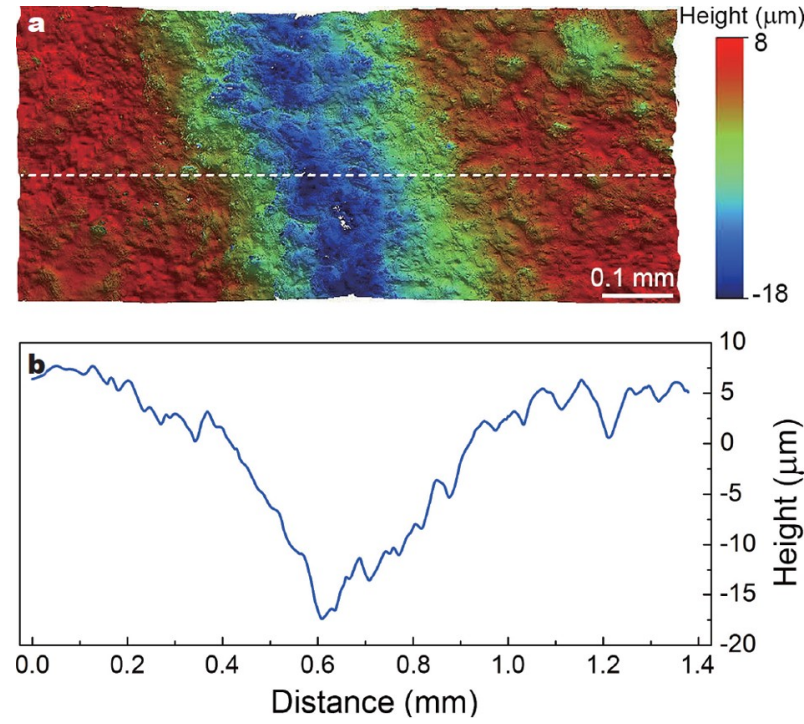

Figure 5 Height profile across a representative $S B$ in the gradient specimen, at the tensile strain of $\sim 0.26$. (a) $2 \mathrm{D}$ contour map; (b) distribution along the dotted white line.
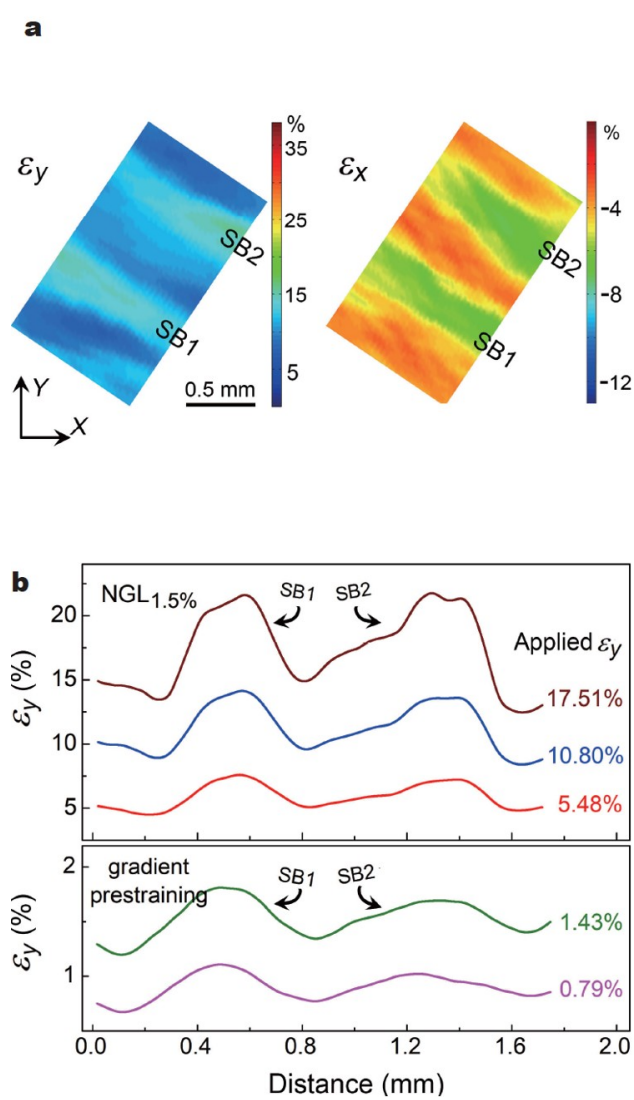

on the preferential strain banding plane $(\theta)$, from

$\left\{\begin{array}{c}\sigma_{\theta}^{\prime}=\sigma_{y} \sin ^{2} \theta, \\ \tau_{\theta}^{\prime}=\sigma_{y} \sin \theta \cos \theta,\end{array}\right.$

to

$\left\{\begin{array}{c}\sigma_{\theta}=\left(\sigma_{y}-\left|\sigma_{y}^{-}\right|\right) \sin ^{2} \theta+\sigma_{x}^{+} \cos ^{2} \theta, \\ \tau_{\theta}=\left(\sigma_{y}-\left|\sigma_{y}^{-}\right|-\sigma_{x}^{+}\right) \sin \theta \cos \theta,\end{array}\right.$

where $\sigma_{y}$ is the externally applied stress. As shown by the Mohr cycles in Fig. 7b, the extra constraints can stabilize the strain banding region by keeping the stress state (the red circle) away from the critical failure boundary (the green curve described by the Ellipse criterion $[38,41]$ ). Therefore, the early SBs in the gradient structure cannot readily propagate across the cross-section to release the applied stress, which keeps the non-strain banding regions high in stress $[19,30,42]$. With increasing applied load, this permits the formation of new stress concentration sites in the regions away from early SBs, thereby providing opportunities to nucleate more SBs
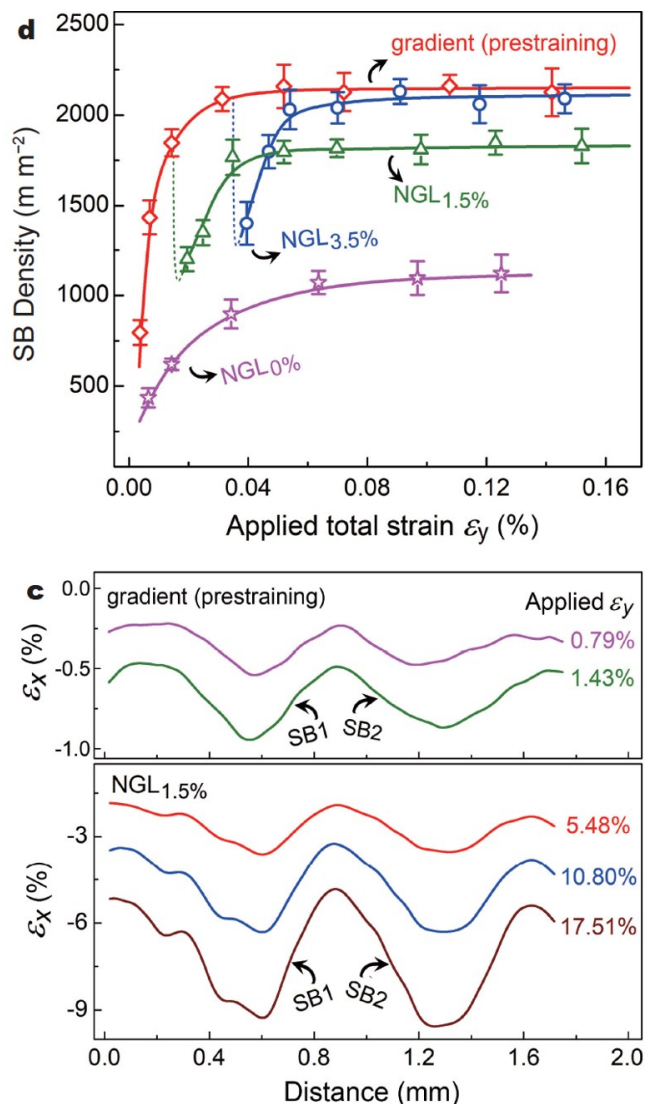

Figure 6 Freestanding $\mathrm{NGL}_{x \%}$ specimen inherited the $\mathrm{SBs}$ formed during prestraining. (a) Enlarged strain contours showing two neighbored representative SBs, i.e., the SB1 and SB2 indicated by blue arrows in Fig. 4b2. (b) and (c) are the statistically averaged distributions of $\varepsilon_{y}$ and $\varepsilon_{x}$ across these two SBs, respectively. (d) Comparison of SB density (the total length of SBs per unit area) in the $\mathrm{NGL}_{x \%}$ and integrated gradient specimens. 
until they dispersively distribute over the whole nanostructured surface layer (Fig. $4 \mathrm{~b}$ and c) [18,36,38].

The low strain hardening capability intrinsic to the nanostructured surface layer is a prerequisite for nucleating SBs [36-39]. The stabilization effect of CG central layer on SBs is the necessary condition for the dispersive nucleation and stable evolution. In other words, it is the hetero-deformation-induced synergistic constraint from the CG central layer that suppresses the quick strain localization and leads to the formation of dispersed stable SBs in the NGL of gradient structure.

\section{Mechanical response controlled by dispersed SBs}

Microstructural observations provide us with information on the crystallographic mechanism behind the stressstrain response of the NGL in the integrated gradient specimen. At the low-strain stage, the highly distorted NS (Fig. 8a) inside the newly nucleated SBs experienced rapid recovery and grain boundary-migration-assisted grain coarsening (Fig. 8b), i.e., deformation-induced softening in SBs $[13,43,44]$. In this regard, the quick multiplication of SBs at the low-strain stage (Fig. 6d) is expected to cause significant strain softening in the nanostructured surface layer. Equilibrium between strain softening in the NS side and strain hardening in the CG side led to the observed flow stress plateau in Fig. 3c. The NGL in the integrated gradient specimen has denser SBs than a freestanding $\mathrm{NGL}_{0 \%}$ specimen (Fig. $6 \mathrm{~d}$ ), which is responsible for the
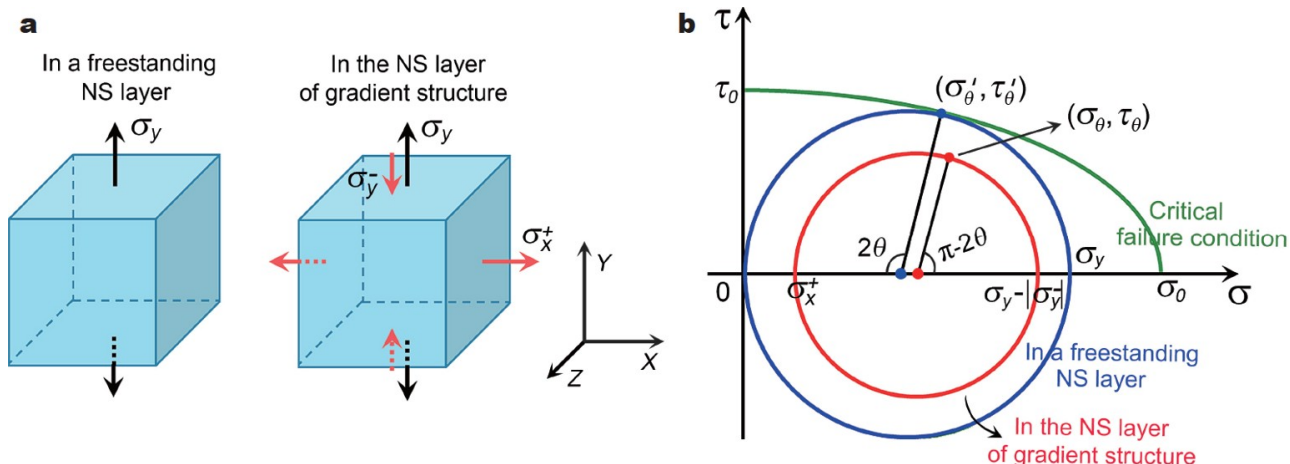

Figure 7 Comparison of the stress states of strain banding region between a freestanding NS layer and the NS layer supported by CG layer in a gradient structure. (a) Stress state of a representative volume element. The red arrow indicates the constraint from CG neighbor. (b) Stress status described by Mohr circles in the $\sigma$ - $\tau$ coordinate. The green curve represents the tensile failure criterion described by $\sigma^{2} / \sigma_{0}^{2}+\tau^{2} / \tau_{0}^{2} \geq 1$ [41], where $\sigma_{0}$ and $\tau_{0}$ are, respectively, the critical normal and shear fracture stresses of NS layer. $Z$ is the specimen thickness direction with mechanical/ microstructural gradient. Since there are free surfaces perpendicular to the $Z$ direction, the stress status are simplified to plane status.
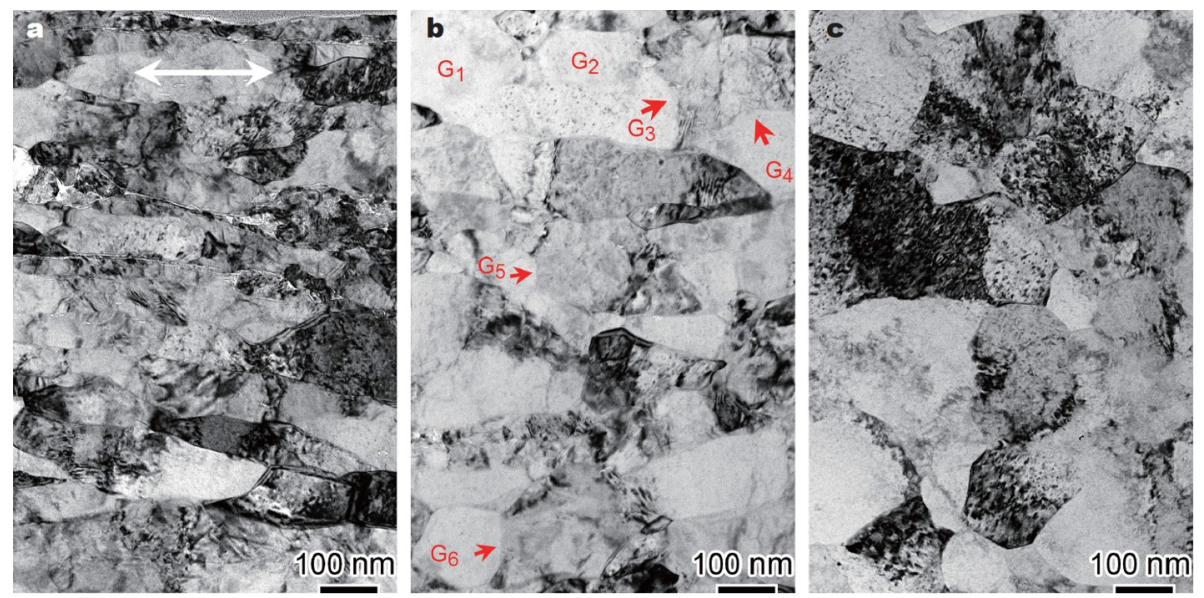

Figure 8 Representative bright-field TEM micrographs, showing microstructure evolution in the strain-banding region of the NGL in the integrated gradient specimen. (a) As-peened NS in the topmost layer. (b) Recovered NS in the SB center with a local $\varepsilon_{y}$ of $\sim 6 \%$. (c) Equiaxed grown-up grains in the SB with a local $\varepsilon_{y}$ of $\sim 37 \%$. White arrow in (a) indicates the direction parallel to the attrition surface. Red arrows in (b) mark the grain boundary migration of coarsening grains $G_{i}$. 
observed lower flow stress (Fig. 3c) and quicker strain hardening rate drop (Fig. 3d) at the low-strain stage.

Importantly, microstructure recovery leaves new room for dislocation accumulation, i.e., regaining strain hardening capability [44-46]. As shown in Fig. 8c, at a higher applied strain some grown-up grains $(\geq 100 \mathrm{~nm})$ in the strain banding region are re-occupied by dislocation entanglements. This suggests that the strain banding regions regained dislocation strengthening after initial recovery and softening [44]. Therefore, denser SBs are expected to help retain a higher strain hardening rate. This is the primary reason why the NGL in the gradient structure displayed transient $\Theta$ up-turning after initial drop and slow $\Theta$ reduction at the high-strain stage (Fig. 3d).

Since the tension-induced grain boundary migration of nanocrystalline is a stress-driven process [47], the lack of $\Theta$ up-turning in the freestanding $\mathrm{NGL}_{0 \%}$ (Fig. 3d) can be attributed to the quick stress release caused by strain localization (Fig. 4a). As analyzed before, it is the stabilization effect of the CG central layer on existing SBs that led to the formation of dispersed stress concentration sites in the nanostructured surface layer. This provides the opportunity to realize extensive microstructure recovery, and then regain dislocation storage. These indicate that the synergistic constraint from CG layer is also the necessary condition for the prevalence of the above crystallographic behaviors.

Note that the regained strain hardening capability in the present NGL is different from the extra strain hardening accompanied by long-range internal stress development $[27,32]$, because the latter originates from strain gradient-dependent geometrically necessary dislocation piling-up around zone (layer) boundaries [7,20,24-26,48]. However, both of them in heterostructure are caused by the deformation incompatibility between heterogeneous zones. The hetero-deformation-induced hardening in heterostructures, therefore, should also include the strain hardening arising from the strain path change of constituent zones, rather than only the extra long-range internal stress hardening [24].

\section{CONCLUSION}

In summary, the stress-strain behavior displayed by the NGL in the tensile testing of a gradient-structured $\mathrm{Ni}$ was experimentally characterized. Significant strain hardening rate $(\Theta)$ up-turning appeared after a flow stress plateau at the low-strain stage, and slow $\Theta$ reduction at the highstrain stage helped with retaining high uniform elongation. Behind such unique constitutive responses, the primary mechanics is that the hetero-deformation- induced synergistic constraint from the CG central layer changed its strain path, from quick coalescence of strain localization to the formation of dense and dispersed stable SBs. The crystallographic mechanism is that the NS in SBs regained strain hardening after initial recovery and grain coarsening. These observations confirm a longpending deformation principle that the hetero-deformation-induced inter-zone constraint modifies the constitutive behavior of constituent zones in heterostructured material.

Received 11 April 2021; accepted 7 May 2021; published online 19 July 2021

1 Wegst UGK, Bai H, Saiz E, et al. Bioinspired structural materials. Nat Mater, 2015, 14: 23-36

2 Wu X, Zhu Y. Heterogeneous materials: A new class of materials with unprecedented mechanical properties. Mater Res Lett, 2017, 5: $527-532$

3 Koyama M, Zhang Z, Wang M, et al. Bone-like crack resistance in hierarchical metastable nanolaminate steels. Science, 2017, 355: 1055-1057

4 Ma E, Zhu T. Towards strength-ductility synergy through the design of heterogeneous nanostructures in metals. Mater Today, 2017, 20: 323-331

5 Lu K. Making strong nanomaterials ductile with gradients. Science, 2014, 345: 1455-1456

6 Zhu Y, Ameyama K, Anderson PM, et al. Heterostructured materials: superior properties from hetero-zone interaction. Mater Res Lett, 2021, 9: 1-31

7 Wu X, Yang M, Yuan F, et al. Heterogeneous lamella structure unites ultrafine-grain strength with coarse-grain ductility. Proc Natl Acad Sci USA, 2015, 112: 14501-14505

8 Wu XL, Jiang P, Chen L, et al. Synergetic strengthening by gradient structure. Mater Res Lett, 2014, 2: 185-191

9 Cheng Z, Zhou H, Lu Q, et al. Extra strengthening and work hardening in gradient nanotwinned metals. Science, 2018, 362: eaau1925

10 Li X, Lu L, Li J, et al. Mechanical properties and deformation mechanisms of gradient nanostructured metals and alloys. Nat Rev Mater, 2020, 5: 706-723

11 Wang YF, Huang CX, Wang MS, et al. Quantifying the synergetic strengthening in gradient material. Scripta Mater, 2018, 150: 22-25

12 Zhang JW, Beyerlein IJ, Han WZ. Hierarchical 3D nanolayered duplex-phase $\mathrm{Zr}$ with high strength, strain hardening, and ductility. Phys Rev Lett, 2019, 122: 255501

13 Fang TH, Li WL, Tao NR, et al. Revealing extraordinary intrinsic tensile plasticity in gradient nano-grained copper. Science, 2011, 331: $1587-1590$

14 Shi P, Ren W, Zheng T, et al. Enhanced strength-ductility synergy in ultrafine-grained eutectic high-entropy alloys by inheriting microstructural lamellae. Nat Commun, 2019, 10: 489

15 Nizolek T, Beyerlein IJ, Mara NA, et al. Tensile behavior and flow stress anisotropy of accumulative roll bonded $\mathrm{Cu}-\mathrm{Nb}$ nanolaminates. Appl Phys Lett, 2016, 108: 051903

16 Wang YF, Wang MS, Fang XT, et al. Extra strengthening in a coarse/ultrafine grained laminate: Role of gradient interfaces. Int J Plast, 2019, 123: 196-207 
17 Wu XL, Jiang P, Chen L, et al. Extraordinary strain hardening by gradient structure. Proc Natl Acad Sci USA, 2014, 111: 7197-7201

18 Huang M, Xu C, Fan G, et al. Role of layered structure in ductility improvement of layered Ti-Al metal composite. Acta Mater, 2018, 153: $235-249$

19 Wang Y, Huang C, Li Z, et al. Shear band stability and uniform elongation of gradient structured material: Role of lateral constraint. Extreme Mech Lett, 2020, 37: 100686

20 Huang CX, Wang YF, Ma XL, et al. Interface affected zone for optimal strength and ductility in heterogeneous laminate. Mater Today, 2018, 21: 713-719

21 Zeng Z, Li X, Xu D, et al. Gradient plasticity in gradient nanograined metals. Extreme Mech Lett, 2016, 8: 213-219

22 Ashby MF. The deformation of plastically non-homogeneous materials. Philos Mag-A J Theor Exp Appl Phys, 1970, 21: 399-424

23 Mughrabi H. Dislocation wall and cell structures and long-range internal stresses in deformed metal crystals. Acta Metall, 1983, 31: 1367-1379

24 Zhu Y, Wu X. Perspective on hetero-deformation induced (HDI) hardening and back stress. Mater Res Lett, 2019, 7: 393-398

25 Ma X, Huang C, Moering J, et al. Mechanical properties of copper/ bronze laminates: Role of interfaces. Acta Mater, 2016, 116: 43-52

26 Park HK, Ameyama K, Yoo J, et al. Additional hardening in harmonic structured materials by strain partitioning and back stress. Mater Res Lett, 2018, 6: 261-267

27 Wang $\mathrm{Y}$, Yang M, Ma X, et al. Improved back stress and synergetic strain hardening in coarse-grain/nanostructure laminates. Mater Sci Eng-A, 2018, 727: 113-118

28 Li J, Zhang Q, Huang R, et al. Towards understanding the structure-property relationships of heterogeneous-structured materials. Scripta Mater, 2020, 186: 304-311

29 Wang YF, Huang CX, Fang XT, et al. Hetero-deformation induced (HDI) hardening does not increase linearly with strain gradient. Scripta Mater, 2020, 174: 19-23

30 Wang Y, Huang C, Li Y, et al. Dense dispersed shear bands in gradient-structured Ni. Int J Plast, 2020, 124: 186-198

31 Feaugas X. On the origin of the tensile flow stress in the stainless steel AISI $316 \mathrm{~L}$ at $300 \mathrm{~K}$ : Back stress and effective stress. Acta Mater, 1999, 47: 3617-3632

32 Yang M, Pan Y, Yuan F, et al. Back stress strengthening and strain hardening in gradient structure. Mater Res Lett, 2016, 4: 145-151

33 He G, Eckert J, Löser W, et al. Novel Ti-base nanostructure-dendrite composite with enhanced plasticity. Nat Mater, 2003, 2: 3337

34 Hays CC, Kim CP, Johnson WL. Microstructure controlled shear band pattern formation and enhanced plasticity of bulk metallic glasses containing in situ formed ductile phase dendrite dispersions. Phys Rev Lett, 2000, 84: 2901-2904

35 Wang YF, Huang CX, He Q, et al. Heterostructure induced dispersive shear bands in heterostructured $\mathrm{Cu}$. Scripta Mater, 2019, 170: $76-80$

36 Ovid'ko IA, Valiev RZ, Zhu YT. Review on superior strength and enhanced ductility of metallic nanomaterials. Prog Mater Sci, 2018, 94: $462-540$

37 Cheng S, Ma E, Wang YM, et al. Tensile properties of in situ consolidated nanocrystalline Cu. Acta Mater, 2005, 53: 1521-1533

38 Zhang P, Qu S, Yang MX, et al. Varying tensile fracture mechanisms of $\mathrm{Cu}$ and $\mathrm{Cu}-\mathrm{Zn}$ alloys with reduced grain size: From necking to shearing instability. Mater Sci Eng-A, 2014, 594: 309320
39 Meyers MA, Mishra A, Benson DJ. Mechanical properties of nanocrystalline materials. Prog Mater Sci, 2006, 51: 427-556

40 Dillamore IL, Roberts JG, Bush AC. Occurrence of shear bands in heavily rolled cubic metals. Metal Sci, 1979, 13: 73-77

41 Zhang ZF, Eckert J. Unified tensile fracture criterion. Phys Rev Lett, 2005, 94: 94301-94304

$42 \mathrm{Wu}$ G, Chan KC, Zhu L, et al. Dual-phase nanostructuring as a route to high-strength magnesium alloys. Nature, 2017, 545: 80-83

43 Zhou X, Li X, Lu K. Size dependence of grain boundary migration in metals under mechanical loading. Phys Rev Lett, 2019, 122 126101

44 Yuan F, Yan D, Sun J, et al. Ductility by shear band delocalization in the nano-layer of gradient structure. Mater Res Lett, 2019, 7: 1217

45 Wang Y, Guo F, He Q, et al. Synergetic deformation-induced extraordinary softening and hardening in gradient copper. Mater Sci Eng-A, 2019, 752: 217-222

46 Fang TH, Tao NR, Lu K. Tension-induced softening and hardening in gradient nanograined surface layer in copper. Scripta Mater, 2014, 77: 17-20

47 Rupert TJ, Gianola DS, Gan Y, et al. Experimental observations of stress-driven grain boundary migration. Science, 2009, 326: 16861690

48 Hasan MN, Liu YF, An XH, et al. Simultaneously enhancing strength and ductility of a high-entropy alloy via gradient hierarchical microstructures. Int J Plast, 2019, 123: 178-195

Acknowledgements This work was supported by the National Natural Science Foundation of China (NSFC, 51931003), China Postdoctoral Science Foundation (2020M680223), the NSFC Basic Science Center Program for "Multiscale Problems in Nonlinear Mechanics" (11988102), the Chinese Academy of Sciences (XDB22040503), and the National Key R\&D Program of China (2017YFA0204403).

Author contributions Wang Y, Huang C and Zhu Y conceived the initial research issues; Wang Y carried out the experiments; Wang Y, Zhu Y and Wu X analyzed the experimental data; Wang Y, Wei Y and Huang C performed theoretical analysis; all authors contributed to discussions; Wang Y, Zhu Y and Huang C wrote the paper.

Conflict of interest The authors declare no conflict of interest.

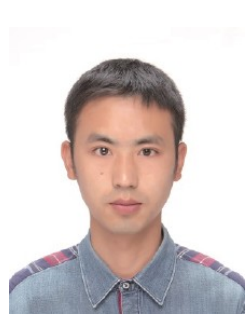

Yanfei Wang received his $\mathrm{PhD}$ degree in solid mechanics in 2020 from Sichuan University. Now he is post-doctoral fellow at Peking University. His current research mainly involves the design principle, deformation physics and corresponding mechanical theory of advanced metallic materials with heterogeneous microstructure. 


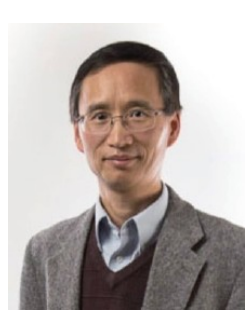

Yuntian Zhu joined the City University of Hong Kong as a Chair Professor in 2020, before which he was a Distinguished Professor at North Carolina State University (2007-2020). He received his $\mathrm{PhD}$ degree in 1994 from the University of Texas at Austin, and worked at Los Alamos National Laboratory in the following thirteen years. His recent research focuses on the deformation mechanisms at dislocation level and mechanical behaviors of heterostructured materials, and nano/ultrafine-grained materials.

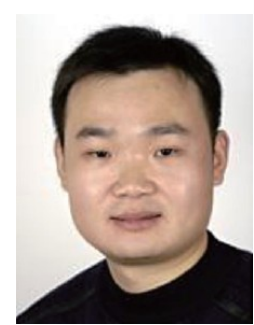

Chongxiang Huang has been a professor since 2010 at Sichuan University. He received his PhD degree in 2006 from the Institute of Metal Research, Chinese Academy of Sciences. He was a Humboldt Scholar at RWTH Aachen University, Germany, from 2008 to 2010 . He has special interest in the deformation fundamentals of heterostructured metallic materials and also in designing metals with superior strength and ductility, including that used in clinical and medical equipment.
异构域交互约束重塑梯度结构成分层的应力-应 变响应

王艳飞 ${ }^{1}$, 朱运田 $^{2^{*}}$, 武晓雷 ${ }^{3}$, 魏悦广 $^{4}$, 黄崇湘 ${ }^{1 *}$

摘要 异构金属材料变形过程中, 成分单元域的应力-应变行为是 理解异构材料变形和强韧化机理的关键科学问题. 本研究以由纳 米结构梯度表层和粗晶芯部层构成的梯度结构为例, 实验观测了 其拉伸变形过程中纳米结构梯度层的应力-应变行为. 结果发现, 纳米结构梯度层表现出区别于其独立变形的独特响应：低应变阶 段, 加工硬化率先急剧降低, 出现流应力平台; 然后, 加工硬化率异 常回复, 流应力升高; 高应变阶段, 加工硬化率缓慢降低, 展现出优 异的均匀延伸率. 该独特响应的力学机制为, 粗晶层约束纳米结构 梯度层的应变局域化, 诱导形成弥散分布且稳定的高密度应变带; 晶体学机制为, 应变带内先发生变形驱动纳米结构回复和晶粒长 大, 然后因位错增殖、累积而硬化. 